И.А. Шафиева ${ }^{1 \bowtie}$, ORCID: 0000-0002-0475-8391, e-mail: ifabtdf@yandex.ru

C.B. Булгакова ${ }^{2,3}$, ORCID: 0000-0003-0027-1786, e-mail: osteoporosis@gmail.com

E.В. Василькова ${ }^{4}$, ORCID: 0000-0002-7671-3969, e-mail: elena.vasilkova@gmail.com

A.B. Шафиева², ORCID: 0000-0002-0865-0687, e-mail: 89.alyona.go123@gmail.com

${ }^{1}$ Клиники Самарского государственного медицинского университета; 443079, Россия, Самара, пр. Карла Маркса, д. $165 Б$

${ }^{2}$ Самарский государственный медицинский университет; 443099, Россия, Самара, ул. Чапаевская, д. 89

3 Министерство здравоохранения Самарской области; 443099, Россия, Самара, ул. Ленинская, д. 73

${ }^{4}$ Клиническая больница «РЖД-медицина»; 443125, Россия, Самара, ул. Ново-Садовая, д. 2226

\title{
Резюме
}

Введение. Остеопороз регистрируется у каждой третьей женщины в возрасте 50 лет и старше. Каждую минуту в стране происходит 7 переломов позвонков, а каждые 5 минут - перелом проксимального отдела бедренной кости. К 2035 г. общее число низкотравматических переломов увеличится с 590 тыс. до 730 тыс. случаев в год.

Цель: изучить влияние МГТ на МПКТ, ткани пародонтального комплекса, суставной синдром у женщин в ПМП.

Материалы и методы. Исследованы 32 женщины в возрасте 50-59 лет в постменопаузе, с хроническом пародонтитом, ПМП ОП, полиостеоартрозом мелких суставов кистей. Контрольная группа ( $\mathrm{n}=14)$ не получалf МГТ (МГТ-). Группа исследования $(\mathrm{n}=18)$ получалf МГТ (МГТ+). Исследовались: перидонтальный индекс, индекс кровоточивости, глубина пародонтального кармана, изучен результат опросника Дрейзера, уровень боли по ВАШ, МПКТ.

Исследования проводились на визите скрининга, через 3, 6 и 12 месяцев.

Результаты. У женщин, получавших менопаузальную терапию, по сравнению с исходными данными и женщинами контрольной группы и через 3 месяца выявлено значимое снижение показателей периодонтального индекса (PI) до 1,2 $\pm 1,05$, индек-

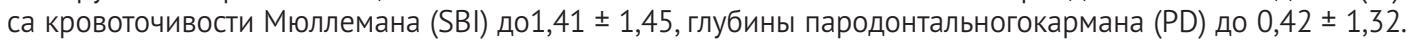

Выявлена тенденция к снижению МПКТ в контрольной группе через 1 год, как в шейке бедра, так и в позвоночнике, на 8,15 и $11,2 \%$.

Заключение. Через 6 месяцев в группе женщин, получающей МГТ, отмечалась стабилизация со стороны пародонтальной области по сравнению с группой контроля. Отмечено уменьшение боли по Шкале ВАШ, улучшение со стороны функционального индекса Драйзера через 3 месяца приема МГТ. Стабилизация положительных эффектов на том же уровне сохранялась в течение 12 месяцев.

Ключевые слова: менопаузальная гормональная терапия, пародонтит, остеопения, минеральная плотность костной ткани, постменопауза

Для цитирования: Шафиева И.А., Булгакова С.В., Василькова Е.В., Шафиева А.В. Влияние эстроген-гестагенной терапии на минеральную костную плотность, состояние тканей пародонтального комплекса, клинических проявлений суставного синдрома у женщин в постменопаузе. Медицинский совет. 2020;(3):139-142. doi: 10.21518/2079-701X-2020-3-139-142.

Конфликт интересов: авторы заявляют об отсутствии конфликта интересов.

\section{The influence of HRT on bone mineral dencity, condition of parodontal complex tissue, clinical implications of articulated syndrome in postmenopausal women}

Irina A. Shafieva1® ${ }^{\circledR}$ ORCID: 0000-0002-0475-8391, e-mail: ifabtdf@yandex.ru.

Svetlana V. Bulgakova2,3, ORCID: 0000-0003-0027-1786, e-mail: osteoporosis@gmail.com

Elena V.Vasilkova ${ }^{4}$, ORCID: 0000-0002-7671-3969, e-mail: elena.vasilkova@gmail.com

Alena V. Shafieva², ORCID: 0000-0002-0865-0687, e-mail: 89.alyona.go123@gmail.com

${ }^{1}$ Clinics of Samara State Medical University; 165B, Karla Marxa Pr., Samara, 443079, Russia

2 Samara State Medical University; 89, Chapaevskaya St., Samara, 443099, Russia

3 Ministry of Health of Samara Region; 73, Leninskaya St., Samara, 443099, Russia

${ }^{4}$ Clinical Hospital "RZD-Medicine”; 222B, Novo-Sadovaya St., Samara, 443125, Russia

\section{Abstract}

Introduction. To evaluate the effect of hormone replacement therapy on periodontal treatment outcomes complex, bone mineral density and articular syndrome in a group of postmenopausal women.

Materials and methods. We carried out an exploration of 32 postmenopausal women aged 50-59 years, with chronic periodontitis, postmenopausal osteoporosis, hand osteoarthritis. The control group $(n=14)$ didn't receive HRT (HRT-). The test group ( $n=18)$ - 
received HRT $(\mathrm{HRT}+$ ).All subjects were examined by recording the following: plaque index (PI), sulcus bleeding index (SBI), periodontal pocket depth (PD), Dreiser`s index, VAS pain score. Measurements were recorded at the baseline, 1 month, 3 months, and 6 monthsю

Results. The significant decline was revealed in all of the values (PI, SBI, PD) in HRT+ compare to HRT- After 6, 12 months, in the periodontal area had been stabilized in the HRT+ group.

$11.2 \%$ reduction was indicated in the lumbar spine and 8,15\% reduction in the femoral neck within the HRT- after 1 year.

The significant decrease was discovered in VAS pain score, Dreiser`s index, painful joints quantity in HRT+.

Conclusions: 12-week HRT was highly effective in periodontal treatment outcomes (significant decrease in PI, SBI, PD after 3 months). BMD of the femoral neck and lumbar spine is reduced within 1 year in patients with MHT- compared with MHT +.In the group of $\mathrm{MHT}+$, compared with the group of MHT- and baseline data, after 3 months there was a significant improvement in the number of painful joints, the level of pain, the Dreiser index, a positive effect persists until the end of the study.

Keywords: hormone replacement therapy, periodontitis, osteopenia, bone mineral density, postmenopause

For citation: Shafieva I.A., Bulgakova S.V., Vasilkova E.V., Shafieva A.V. The influence of HRT on bone mineral dencity, condition of parodontal complex tissue, clinical implications of articulated syndrome in postmenopausal women. Meditsinskiy sovet $=$ Medical Council. 2020;(3):139-142. (In Russ.) doi: 10.21518/2079-701X-2020-3-139-142.

Conflict of interest: The authors declare no conflict of interest.

\section{ВВЕДЕНИЕ}

В связи с увеличением продолжительности жизни в современном обществе возрос интерес к проблемам здоровья женщин в постменопаузе [1]. Возраст наступления менопаузы в настоящее время остается относительно стабильным и составляет в 45-50 лет [2]. Если учесть, что в этот период жизни женщина, как правило, ведет активную социальную жизнь, то становятся очевидными проблемы эстрогенного дефицита для большей части населения нашей планеты. Дистрофические изменения, вызванные прекращением или снижением функции половых желез у женщин, наблюдаются в костной системе, в ткани пародонта, в суставах и околосуставных тканях [3-5]. Остеопороз регистрируется у каждой третьей женщины в возрасте 50 лет и старше. Каждую минуту в стране происходит 7 переломов позвонков, а каждые 5 минут - перелом проксимального отдела бедренной кости. К 2035 г. общее число низкотравматических переломов увеличится с 590 тыс. до 730 тыс. случаев в год [6]. Установлено, что у пациентов с остеопорозом хронический генерализованный пародонтит тяжелой степени отмечается чаще, чем у пациентов без такового (41,5 и 12,5\% соответственно) [7].

В возрасте 50-70 лет остеоартроз кистей диагностируется у 70\% населения, а рентгенологические признаки встречаются в $90 \%$ случаев [3, 8].

Таким образом, актуальность изучения проблем патогенеза, диагностики и лечения постменопаузальных нарушений не вызывает сомнений, а решение указанных вопросов требует детальных исследований.

Цель: изучить влияние непрерывной эстроген-гестагенной терапии (препарат Фемостон ${ }^{\circledR}$ Конти) на минеральную костную плотность, состояние тканей пародонтального комплекса, клинические проявления суставного синдрома у женщин в постменопаузе.

Для реализации этой цели были поставлены задачи:

1. Выявить динамику изменений тканей пародонтального комплекса у женщин, получающих эстроген-гестагенную терапию и не получающих МГТ, исходно, через 3, 6 и 12 месяцев.

2. Изучить в сравнительном аспекте показатели МПКТ в шейке бедра и поясничном отделе позвоночника у женщин, получающих эстроген-гестагенную терапию и не получающих МГТ, исходно и через 12 месяцев.

3. Изучить влияние МГТ на суставной синдром (как клинический симптомокомплекс, обусловленный поражением анатомических структур суставов при полиостеоартрозе 1-2 рентгенологической стадии) у женщин в постменопаузе.

\section{МАТЕРИАЛЫ И МЕТОДЫ}

В исследование включены 32 пациентки в возрасте 50-59 лет (средний возраст 55,4 \pm 4,3 лет) в постменопаузе, с хроническим пародонтитом, постменопаузальной остеопенией, полиостеоартрозом мелких суставов кистей 1-2 рентгенологической стадии с болевым синдромом. Пациентки были разделены на две группы. Контрольную группу (n=14) составили женщины, не получающие МГТ (МГТ-). Группа исследования ( $\mathrm{n}=18)$ состояла из женщин, которые получали МГТ (МГТ+) (17 $\beta$-эстрадиол и дидрогестерон препарат Фемостон ${ }^{\circledR}$ Конти) в течение 12 месяцев от начала исследования. Группа исследования и контрольная группа были сопоставимы по всем собранным гинекологическим параметрам. Ни одна из исследуемых не получала МГТ ранее. Средний возраст первых менструаций составил 13 лет, средний возраст наступления менопаузы - 48 лет. Естественная менопауза наступила у 88\% исследованных женщин, хирургическая менопауза - у $12 \%$. Средняя продолжительность постменопаузы составляла $3,2 \pm 0,9$ лет.

Пациентки обеих групп были исследованы: периодонтальный индекс Rassell (PI), индекс кровоточивости Мюллемана (SBI), глубина пародонтального кармана (PD).

Исследования проводились на визите скрининга и далее через 3, 6 и 12 месяцев.

Определена МПКТ при помощи двухэнергетической рентгеновской абсорбциометрии на аппарате HOLOGIC по шейке бедра и поясничному отделу позвоночника в начале исследования и через 12 месяцев [9].

Все пациентки предъявляли жалобы на боли в мелких суставах кистей, им были выполнены рентгенологическое исследование кистей в прямой проекции и выявлен полиостеоартроз 1-2 рентгенологической стадии. 
Bсе пациентки были протестированы при помощи опросника Дрейзера. Уровень боли рассчитывали по шкале ВАШ [10-15].

Расчеты проводили на персональном компьютере с использованием пакета программ «StatisticaforWindows» русская версия 6.1.478 (StatSoftInc., Tulsa, USA), а также MedCalc ${ }^{\circledR}$ 10.2.0.0 (MedCalcSoftwarebvba, Mariakerke, Belgium).

\section{РЕЗУЛЬТАТЫ ИССЛЕДОВАНИЯ}

Динамика изменений тканей пародонтального комплекса у женщин, получающих эстроген-гестагенную терапию и не получающих МГТ, исходно, через 3, 6 и 12 месяцев представлена на рис.

В группе МГТ+ по сравнению с группой МГТ- и исходными данными через 3 месяца имеет место значимое снижение показателей PI, SBI, PD ( $p<0,05)$. Через 6 месяцев в группе, получающей МГТ, отмечалась стабилизация со стороны пародонтальной области по сравнению с группой контроля, что выражалось в тенденции к дальнейшему снижению периодонтального индекса, индекса кровоточивости, уменьшении глубины пародонтального кармана.

В группе женщин, получающей МГТ, не наблюдалось прогрессирования пародонтальных нарушений. Через 12 месяцев периодонтальный индекс, индекс кровоточи- вости, глубина пародонтальных карманов у этих женщин были на том же уровне, что и через 3 и 6 месяцев приема МГТ. Следует отметить, что состояние пародонта в контрольной группе несколько ухудшилось.

Показатели МПКТ в шейке бедра и поясничном отделе позвоночника у женщин, получающих эстроген-гестагенную терапию и не получающих МГТ, исходно и через 12 месяцев представлены в табл. 1.

Анализ полученных данных не выявил значимых различий в состоянии костной массы по Т-критерию шейки бедра и поясничного отдела позвоночника. При этом отмечена тенденция к снижению МПКТ, как в шейке бедренной кости, так и в поясничном отделе позвоночника у пациентов, не получающих МГТ, на 8,15 и 11,2\% соответственно.

Влияние МГТ на суставной синдром (как клинический симптомокомплекс, обусловленный поражением анатомических структур суставов при полиостеоартрозе 1-2 рентгенологической стадии) у женщин в постменопаузе представлено на табл 2.

Анализ данных показал, что количество болезненных суставов значимо не изменилось. Следует отметить уменьшение боли по Шкале ВАШ, улучшение со стороны функционального индекса Драйзера через 3 месяца приема МГТ, которые оставались на том же уровне в течение 12 месяцев.

Рисунок. Динамика изменений тканей пародонтального комплекса исходно, через 3, 6 и 12 месяцев

Figure. Dynamics of tissue changes in the periodontal complex at baseline, 3, 6, 12 months
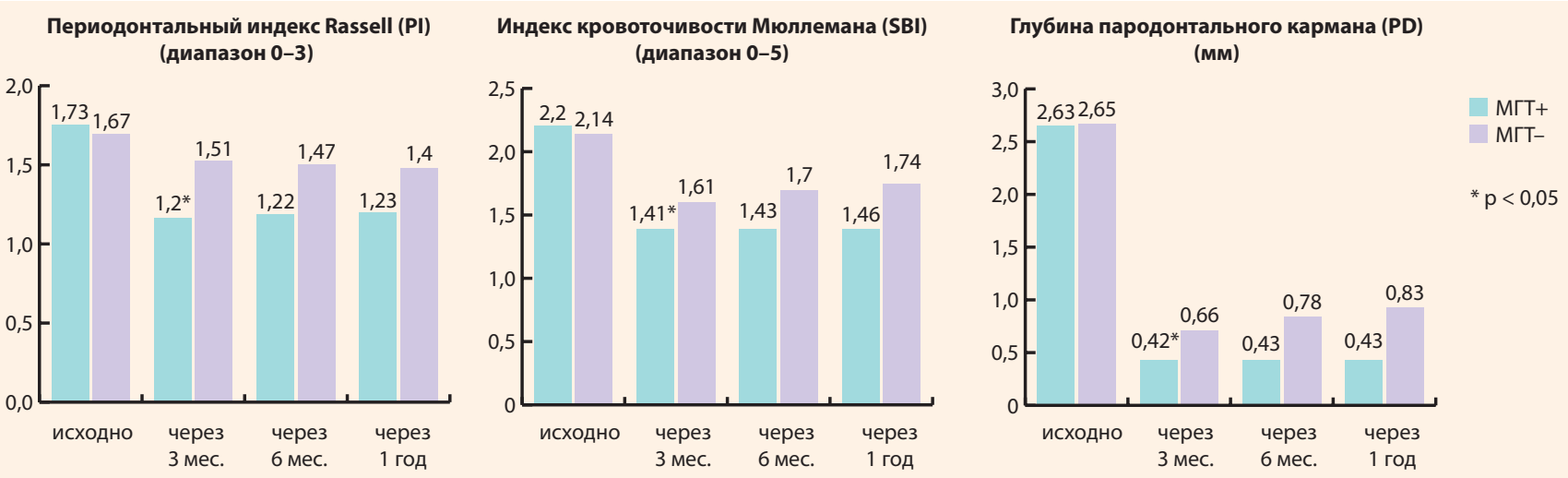

- Таблица 1. Сравнение исходных показателей МПКТ и через 12 месяцев в обеих группах

- Table 1. Comparison of BMD at baseline and after 12 months in both groups

\begin{tabular}{l|c|c|c|c}
\multicolumn{1}{c|}{ Состояние КМ } & МГТ- исходно & МГТ+ исходно & МГТ-через 12 месяцев & МГТ+ через 12 месяцев \\
\hline Т-критерий (среднее значение) по шейке бедра & $-1,213 \pm 0,360$ & $-1,282 \pm 0,342$ & $-1,311 \pm 0,362, \mathrm{p}>0,05$ & $-1,281 \pm 0,344, \mathrm{p}>0,05$ \\
\hline $\begin{array}{l}\text { Т-критерий (среднее значение) } \\
\text { по поясничному отделу позвоночника }\end{array}$ & $-2,124 \pm 0,364$ & $-2,134 \pm 0,521$ & $-2,2361 \pm 0,363$ & $-2,135 \pm 0,520$
\end{tabular}

Таблица 2. Влияние МГТ на суставной синдром у женщин в постменопаузе

Table 2. Effect of MHT on the joint syndrome in postmenopausal women

\begin{tabular}{|c|c|c|c|c|c|c|c|c|}
\hline 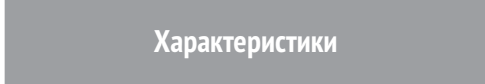 & $\begin{array}{c}\text { МГТ+ } \\
\text { исходно }\end{array}$ & $\begin{array}{c}\text { МГТ- } \\
\text { исходно }\end{array}$ & $\begin{array}{c}\text { МГТ+ } \\
\text { через } 3 \text { мес }\end{array}$ & $\begin{array}{c}\text { МГТ- } \\
\text { через } 3 \text { мес }\end{array}$ & $\begin{array}{c}\text { МГТ+ } \\
\text { через } 6 \text { мeс }\end{array}$ & $\begin{array}{c}\text { МГТ- } \\
\text { через } 6 \text { мес }\end{array}$ & $\begin{array}{c}\text { МГТ+ } \\
\text { через } 1 \text { год }\end{array}$ & $\begin{array}{c}\text { МГТ- } \\
\text { через } 1 \text { год }\end{array}$ \\
\hline Количество боле & & $3,7 \pm 3,1$ & & & & & $2,08 \pm 3,9$ & \\
\hline Функциональный индекс Драйзера & $11,3 \pm 3,8$ & $12,3 \pm 4,8$ & $8,34 \pm 3,9$ & $12,2 \pm 2,2$ & $8,33 \pm 4,9$ & $12,5 \pm 5,3$ & $8,32 \pm 5,4$ & $12,6 \pm 3,3$ \\
\hline лобальная спонтанная боль по ВАШ (мм) & $55,8 \pm 14,4$ & $53,4 \pm 15,6$ & $34,47 \pm 12,5$ & $52,4 \pm 10,3$ & $34,46 \pm 16,3$ & $52,5 \pm 11,5$ & $34,54 \pm 13,3$ & $53 \pm 12,6$ \\
\hline
\end{tabular}




\section{ЗАКЛЮЧЕНИЕ}

Наш опыт показал, что через 6 месяцев в группе женщин, получающей МГТ, отмечалась стабилизация со стороны пародонтальной области, по сравнению с группой контроля. Это выражалось в тенденции к дальнейшему снижению периодонтального индекса, индекса кровоточивости, уменьшении глубины пародонтального кармана. В группе женщин, получающей МГТ, не наблюдалось прогрессирования пародонтальных нарушений. Через 12 месяцев периодонтальный индекс, индекс кровоточивости, глубина пародонтальных карманов у этих женщин были на том же уровне, что и через 3 и 6 месяцев приема МГТ. Также отмечено уменьшение боли по Шкале ВАШ, улучшение со стороны функционального индекса Драйзера через 3 месяца приема МГТ и стабилизация положительных эффектов на том же уровне в течение 12 месяцев.

Следует отметить, что в контрольной группе состояние пародонта несколько ухудшилось, выявлено снижение МПКТ в шейке бедренной кости и в поясничном отделе позвоночника на 8,15 и 11,2\% соответственно.

Поступила / Received 10.03.2020

Поступила после рецензирования / Revised 24.03.2020

Принята в печать / Accepted 25.03.2020

\section{Список литературы / References}

1. Gold E.B. The timing of the age at which natural menopause occurs. Obstet Gynecol Clin North Am. 2011;38(3):425-440. doi: 10.1016/j.ogc.2011.05.002

2. Ceylan B., Özerdoğan N. Factors affecting age of onset of menopause and determination of quality of life in menopause. Turk J Obstet Gynecol. 2015;12(1):43-49. doi: $10.4274 /$ tjod.79836.

3. Cekici A., Baser U., Isik G., Akhan S.E., Issever H., Onan U. Periodontal treatment outcomes in post menopausal women receiving hormone replacement therapy. J Istanb Univ Fac Dent. 2015;49(3):39-44. doi: 10.17096/jiufd.61637.

4. Pizzo G., Guiglia R., Licata M.E., Pizzo I., Davis J.M., Giuliana G. Effect of hormone replacement therapy (HRT) on periodontal status of postmenopausal women. Med Sci Monit. 2011;17(4):PH23-PH27. doi: 10.12659/MSM.881700.

5. Ran S.Y., Yu Q., Chen Y., Lin S.Q. Prevention of postmenopausal osteoporosis in Chinese women: A 5-year, double-blind, randomized, parallel placebo-controlled study. Climacteric. 2017;20:391-396. doi: 10.1080/13697137.2017.1325459.

6. Лесняк О.М., Баранова И.А., Белова К.Ю., Гладкова Е.Н., Евстигнеева Л.П., Ершова О.Б. и соавт. Остеопороз в российской федерации: эпидемиология, медико-социальные и экономические аспекты проблемы (обзор литературы). Травматология и ортопедия России. 2018;24(1):155-168. doi: 10.21823/23112905-2018-24-1-155-168.

Lesnyak O.M., Baranova I.A., Belova K.Y., Gladkova E.N., Evstigneeva L.P., Ershova O.B. et al. Osteoporosis in russian federation: epidemiology, socio-medical and economical aspects (review). Travmatologiya $i$ ortopediya Rossii $=$ Traumatology and Orthopedics of Russia. 2018;24(1):155-168. (In Russ.) doi: 10.21823/2311-29052018-24-1-155-168.

7. Намханов В.В. Влияние потери минеральной плотности костной ткани на пародонтальный статус пациентов. Вестник Бурятского Государственного Университета. Медицина и фармация. 2014;(12):93-96. Режим доступа: https:// elibrary.ru/item.asp?id=22532195.

Namkhanov V.V. Impact of the loss of mineНамханов В.В. Влияние потери минеральной плотности костной ткани на пародонтальный статус пациентов. Вестник Бурятского Государственного Университета. Медицина и фармация. 2014;(12):93-96. Режим доступа: https://elibrary.ru/item.asp?id=22532195.
Namkhanov V.V. Impact of the loss of mineral density of bone tissue on the periodontal status of patients. Vestnik Buryatskogo Gosudarstvennogo Universiteta. Meditsina i farmatsiya = Bulletin of Banzarov Buryat State University. Medicine and Pharmacy. 2014;(12):93-96. (In Russ.) Available at: https://elibrary.ru/item.asp?id=22532195.

8. Xue W., Deng Y., Wang Y.F., Sun AJ. Effect of half-dose and standard-dose conjugated equine estrogens combined with natural progesterone or dydrogesterone on components of metabolic syndrome in healthy postmenopausal women: A randomized controlled trial. Chin Med J (Eng). 2016;129:2773-2779.doi: 10.4103/0366-6999.194646.

9. Posch M., Schranz A., Lener M. et al. Effectiveness of a Mini-Trampoline Training Program on Balance and Functional Mobility, Gait Performance, Strength, Fear of Falling and Bone Mineral Density in Older Women with Osteopenia. Clin Interv Aging. 2019:14:2281-2293. doi: 10.2147/CIA.S230008.

10. Almeida M., Laurent M.R., Dubois V. et al. Estrogens and androgens in skeletal physiology and pathophysiology. Physiol Rev. 2017;97(1):135-187. doi: 10.1152/ physrev.00033.2015.

11. Kim MJ., Cho J., Ahn Y., Yim G., Park H.Y. Association between physical activity and menopausal symptoms in perimenopausal women. BMC Womens Health. 2014;14:122. doi: 10.1186/1472-6874-14-122

12. Cosman F., de Beur SJ., LeBoff M.S., Lewiecki E.M., Tanner B., Randall S. Clinician's guide to prevention and treatment of osteoporosis. Osteoporos Int. 2014;25(10):2359-2381. doi: 10.1007/s00198-014-2794-2.

13. Svedboum A., Hernlund E., Ivergard M., Compston J., Cooper C Stenmark J. Osteoporosis in the European Union: a compendium of country-specific reports. Arch Osteoporos. 2013;8:137. doi: 10.1007/s11657-013-0137-0.

14. Rodríguez-Landa J.F., Puga-Olguín A., Germán-Ponciano LJ., García-Ríos R.I., SoriaFregozo C. Anxiety in Natural and Surgical Menopause - Physiologic and Therapeutic Bases. In: Durbano F. A Fresh Look at Anxiety Disorders. London: IntechOpen; 2015, pp. 173-196. doi: 10.5772/60621

15. Kannus P., Sievänen H., Palvanen M., Järvinen T., Parkkari J. Prevention of falls and consequent injuries in elderly people. Lancet. 2005;366(9500):1885-1893. doi: 10.1016/S0140-6736(05)67604-0.

\section{Информация об авторах:}

Шафиева Ирина Алексеевна, к.м.н., врач-ревматолог высшей квалификационной категории, заведующая отделением эндокринологии и остеопороза, Федеральное государственное бюджетное образовательное учреждение высшего образования «Клиники Самарского государственного медицинского университета»; 443079, Россия, Самара, пр. Карла Маркса, д. 165Б; e-mail: ifabtdf@yandex.ru

Булгакова Светлана Викторовна, д.м.Н., доцент, заведующая кафедрой гериатрии и возрастной эндокринологии, Федеральное государственное бюджетное образовательное учреждение высшего образования «Самарский государственный медицинский университет» Министерства здравоохранения Российской Федерации; 443099, Россия, Самара, ул. Чапаевская, д. 89; главный внештатный специалист по гериатрии, Министерство здравоохранения Самарской области; 443010, Россия, Самара, ул. Ленинская, д. 73; e-mail: osteoporosis@gmail.com

Василькова Елена Валерьевна, к.м.н., врач акушер-гинеколог, Частное учреждение здравоохранения «Клиническая больница «РжДмедицина»; 443125, Россия, Самара, ул. Ново-Садовая, д. 222б; e-mail: elena.vasilkova@gmail.com

Шафиева Алёна Витальевна, студентка 5-го курса лечебного факультета, Федеральное государственное бюджетное образовательное учреждение высшего образования «Самарский государственный медицинский университет» Министерства здравоохранения Российской Федерации; 443099, Россия, Самара, ул. Чапаевская, д. 89; e-mail: 89.alyona.go123@gmail.com

\section{Information about the authors:}

Irina A. Shafieva, Cand. of Sci. (Med.), Revmatologist of the Highest Qualification Grade, Head of Endocrinology and Osteoporosis Department, Federal State Budgetary Educational Institution of Higher Education "Clinics of Samara State Medical University"; 3 , Karla Marxa St., Samara, 443079, Russia; e-mail: ifabtdf@yandex.ru

Svetlana V. Bulgakova, Dr. of Sci. (Med.), Associate Professor, Head of Chair for Geriatrics and Age Endocrinology, Federal State Budgetary Educational Institution of Higher Education "Samara State Medical University" of the Ministry of Health of the Russian Federation; 89, Chapaevskaya St., Samara, 443099, Russia; Chief External Expert in Geriatrics, Ministry of Health of the Samara Region; 73, Leninskaya St., Samara, 443010,Russia; e-mail: osteoporosis@gmail.com

Elena V. Vasilkova, Cand. of Sci. (Med.), Obstetrician-Gynecologist, Private Healthcare Institution "Central Clinical Hospital «RZD-Medicine»: 222B, Novo-Sadovaya St., Samara, 443125, Russia; e-mail: elena.vasilkova@gmail.com

Alena V. Shafieva, a fifth year student, General Medicine Faculty, Federal State Budgetary Educational Institution of Higher Education “Samara State Medical University" of the Ministry of Health of the Russian Federation; 89, Chapaevskaya St., Samara, 443099, Russia; e-mail: 89.alyona.go123@gmail.com 